\title{
Efficient Route to Label Mesenchymal Stromal Cell-Derived Extracellular Vesicles
}

\author{
Diego Alberti, ${ }^{\dagger}$ Cristina Grange, ${ }^{\ddagger}$ Stefano Porta, ${ }^{\dagger}$ Silvio Aime, ${ }^{\dagger}$ Lorenzo Tei, ${ }^{\S}$ \\ and Simonetta Geninatti Crich ${ }^{*} \dagger \odot$ \\ ${ }^{\dagger}$ Department of Molecular Biotechnology and Health Science, University of Turin, Via Nizza 52, 10126 Torino, Italy \\ ${ }^{\ddagger}$ Department of Medical Sciences, University of Turin, Corso Dogliotti 14, 10126 Torino, Italy \\ ${ }^{\S}$ Department of Science and Technological Innovation, University of Piemonte Orientale, Viale T. Michel 11, 15121 Alessandria, \\ Italy
}

\section{Supporting Information}

ABSTRACT: Recent research results report that extracellular vesicles (EVs) have a central role in both physiological and pathological processes involving intercellular communication. Herein, a simple EVs labeling procedure based on the metabolic labeling of secreting cells (mesenchymal stroma cells, MSCs) with a fluorescein-containing bio-orthogonal dye is described. This procedure was carried out by incubating cells for $72 \mathrm{~h}$ with tetraacetylated $\mathrm{N}$-azidoacetyl-D-mannosamine $\left(\mathrm{Ac}_{4} \mathrm{ManNAz}\right)$, a modified sugar containing an azido group that, upon incorporation on the external surface of the cytoplasmatic cell membrane, is specifically conjugated with cyclooctyne-modified fluorescein isothiocyanate (ADIBOFITC). MSCs released fluorescent EVs did not need any

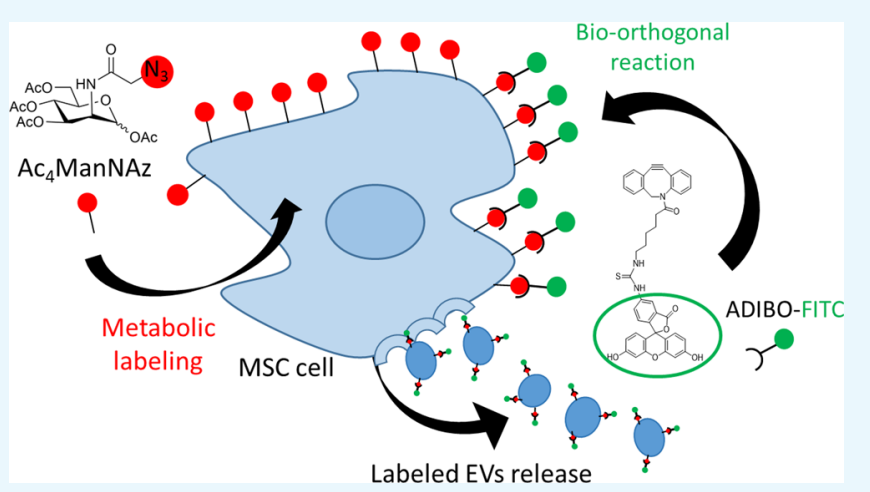
further purification. Finally, cellular uptake and tracking of the fluorescein-labeled EVs were successfully assessed by targeting experiments with MSCs. The method appears of general applicability and it may be very useful opening new horizon on diagnostic and therapeutic protocols exploiting EVs.

\section{INTRODUCTION}

It is now well established that extracellular vesicles (EVs) are a powerful tool for intercellular communication. ${ }^{1}$ They consist of a heterogeneous population of double layers membrane fragments that are released by most cell types. ${ }^{2}$ EVs can carry a payload made of molecules such as proteins, lipids, and nucleic acids that are uptaken by target cells, influencing their fate. $^{3-6}$ In particular, a pro-regenerative ability has been ascribed to EVs released by stem cells, that act by stimulating recipient cell proliferation, inhibiting apoptosis, and favoring immune escape. ${ }^{7-10}$ The use of stem cell derived EVs is currently under intense scrutiny, as a novel putative therapeutic option. In fact, it has been reported that EVs released by mesenchymal stroma cells (MSCs) resulted highly efficient in the treatment of several pathologies in preclinical experimental models. ${ }^{11,12}$ A very promising characteristics of EVs relies in their ability to transport genetic information, as well as drugs and proteins, to target cells. In addition, EVs possess unique properties including (i) enhanced passive targeting due to their small size, (ii) excellent biological acceptance due to their endogenous nature, and (iii) ability to cross biological barriers. Their natural potential to transfer biological materials from one cell to another can be enhanced or customized to enable a wide range of therapeutic applications through the introduc- tion of molecules that facilitate targeting, uptake, and loading of EVs. To improve the use of MSC EVs, it is essential to track EV biodistribution in vivo. Moreover, conjugation of imaging probes to the surface of EVs that allows their not-invasive detection is an important challenge. Currently, labeling strategies used are based essentially on the use of highly lipophilic fluorescent dyes, able to stain artificial and biological membranes thanks to the presence of lipophilic long-chains functionalized with cyanine moieties (i.e., PKH, DiD, DiI, and R18). ${ }^{13-15}$ The aliphatic chain rapidly intercalates into the EVs lipid bilayer, thus providing the fluorescent labeling of the particles. Unfortunately, the protocols used to remove dye aggregates nonbound to EVs lipidic membrane are often insufficient, leading to the occurrence of many false positive results. $^{16,17}$ For these reasons, it appears useful to find alternative, easy, and fast labeling procedures that avoid the unspecific incorporation into the membrane of both EVs and MSCs as in the case of lipophilic dyes. Herein, we report our results on an EV labeling procedure with fluorescent dyes based on the bio-orthogonal copper-free click chemistry

Received: $\quad$ May 4, 2018

Accepted: July 6, 2018

Published: July 19, 2018 


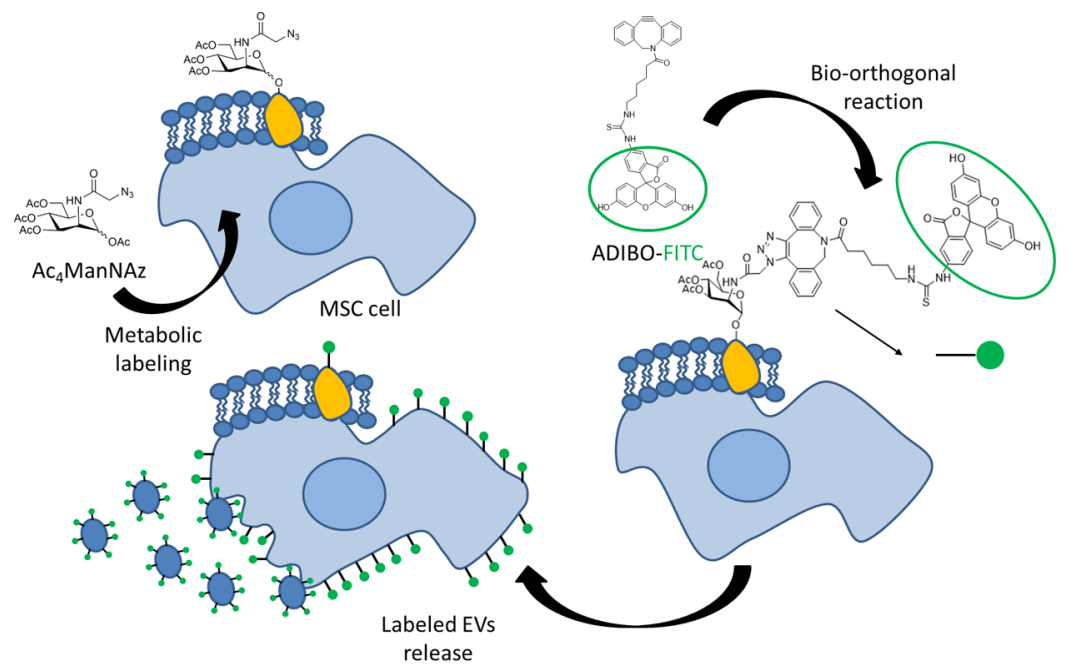

Figure 1. Description of the bio-orthogonal labeling procedure of EVs.

approach. In recent years, the bio-orthogonal approach has attracted considerable attention for cell labeling and/or tracking because of its high specificity and low toxicity to cells. ${ }^{18-20}$ Among the possible bio-orthogonal approaches, the rapid, biocompatible, and specific chemical reaction between the functionalized dibenzocyclooctyne and azido-sugars generated on the cell surface by metabolic glycoengineering has shown great potential. ${ }^{21-23}$ Thus, applying this approach, cancer cells labeling strategies and targeted delivery of nanoparticles have been recently developed and validated also in vivo. ${ }^{24-28}$

Thus, first, the metabolic labeling of MSCs with tetraacetylated $\mathrm{N}$-azidoacetyl-D-mannosamine ( $\mathrm{Ac}_{4} \mathrm{ManNAz}$ ) was carried out to generate unnatural azide groups on the surface of the cells. This task was accomplished by growing MSC cells in the presence of the modified sugar, and then the azide groups specifically and efficiently reacted with fluorescein conjugated aza-dibenzocyclooctyne (ADIBO-FITC) (Figure 1) via the $\mathrm{Cu}$-free 1,3-dipolar cycloaddition reaction. A similar bio-orthogonal approach has been very recently used by Lee et $\mathrm{al}^{29}$ to label exosomes isolated by breast cancer cells (MCF7 and MDA-MB-231) with a Cy3 fluorescent dye. However, stem cells and MSCs used in this work possess completely different characteristics compared with tumor cells, in terms of metabolism, size, growth rate, and phenotypic markers. In addition, MSCs may have different extent of extracellular glycan formation with respect to tumor cells and different amount of exosomes secreted.

\section{RESULTS AND DISCUSSION}

Mesenchymal Stroma Cells Labeling. To expose azide groups on the surface of MSCs, the cells were incubated in the presence of a solution of $\mathrm{Ac}_{4} \mathrm{ManNAz}(50 \mu \mathrm{M}$ in $0.25 \mathrm{v} / \mathrm{v} \%$ ethanol in MSC basal medium) for $72 \mathrm{~h}$ at $37{ }^{\circ} \mathrm{C}\left(5 \% \mathrm{CO}_{2}\right)$. Azide-modified sugars were incorporated through existing biosynthetic pathways both onto the cell surface and on internal glycans. After incorporation, the unnatural azide group is available for reaction with the stable ADIBO-FITC (Figure $1)$. Thus, a further incubation of $30^{\prime}$ at $37^{\circ} \mathrm{C}$ in the presence of $40 \mu \mathrm{M}$ ADIBO-FITC $(0.2 \mathrm{v} / \mathrm{v} \%$ ethanol in MSC basal medium) was carried out to allow the $\mathrm{Cu}$-free click chemistry reaction to occur on cell surface. Then, after washing, the fluorescent labeling on MSCs was assessed by flow cytometry.
As reported in Figure 2A, the fluorescence signal of MSCs treated with $\mathrm{Ac}_{4} \mathrm{ManNAz}$ and ADIBO-FITC was much higher

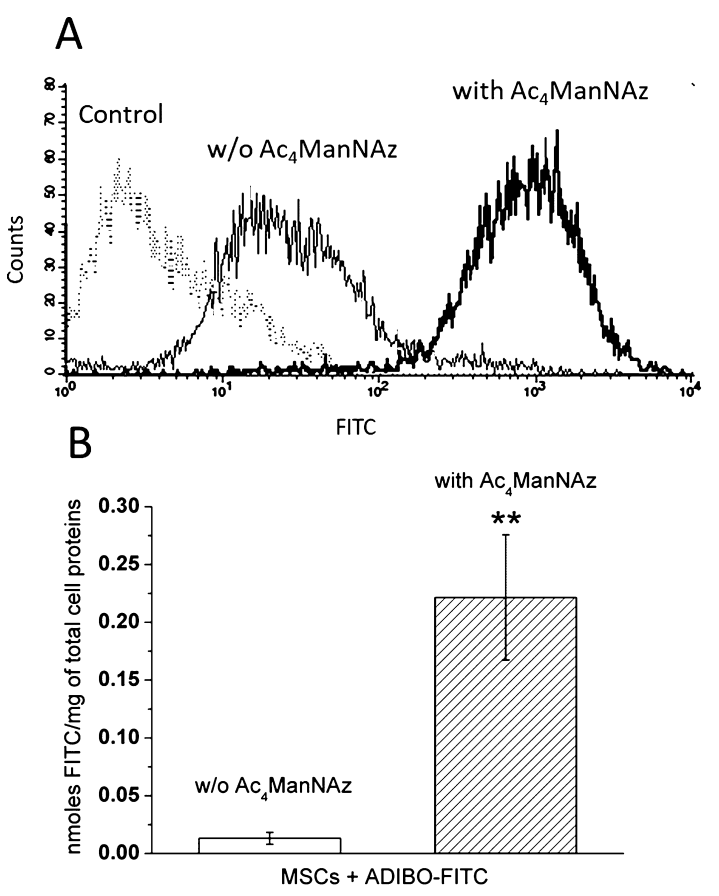

Figure 2. Representative cytofluorimetric (A) and spectrofluorimetric (B) analysis of MSCs labeled with ADIBO-FITC pre-incubated in the presence or in the absence of $\mathrm{Ac}_{4} \mathrm{ManNAz}$. Fluorescence values measured on cell lysates (upon treatment with triton 0.1\%) were normalized to the total cell protein content determined by Bradford assay. Data are the mean $\pm \mathrm{SE}$ of seven different experiments. $* * p=$ 0.0024 , Student $t$-test.

with respect to control cells treated only with ADIBO-FITC, confirming the successful labeling of the cells via the click reaction. Different aliquots of the same cell samples were then lysated and analyzed by spectrofluorimetry (Figure 2B). The amount of FITC bound to MSCs was quantified based on a FITC standard curve, and it was normalized to the total protein content of cells determined by the Bradford method. Figure $2 \mathrm{~B}$ confirms the results obtained by fluorescence activated cell sorting (FACS) analysis, thus evidencing the 
absence of a nonspecific binding of ADIBO-FITC to the cell cytosolic membrane in comparison with $\mathrm{Ac}_{4} \mathrm{ManNAz}$-treated cells. This clearly demonstrated that the amount of exposed azide groups was sufficient to allow the detection of labeled MSCs. No advantages were observed when different concentrations of ADIBO-FITC (15 and $60 \mu \mathrm{M})$ were used (Supporting Information Figure S1).

To assess whether MSC labeling was maintained over time, cells previously treated with $\mathrm{Ac}_{4} \mathrm{ManNAz}$ and $\mathrm{ADIBO}-\mathrm{FITC}$ were kept in culture for additional 5 days. FACS analysis of MSCs was performed at day 3 and day 5 . Figure 3 shows that 5

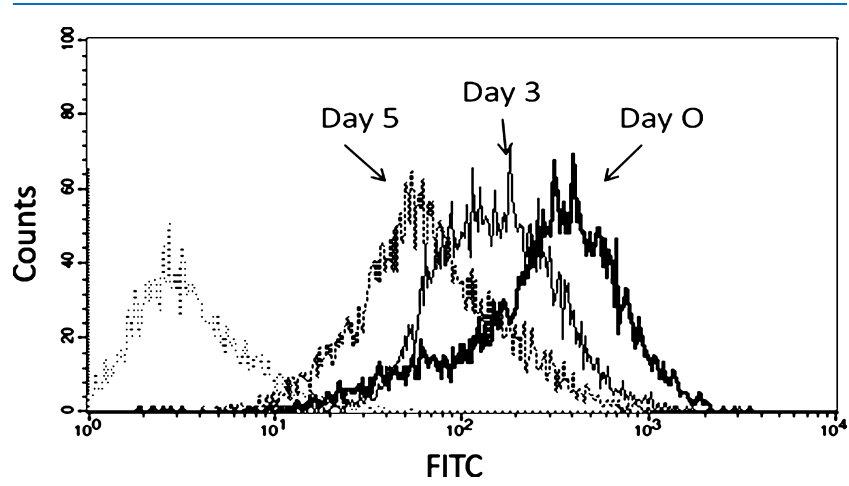

Figure 3. Representative cytofluorimetric analysis of fluorescence maintenance by MSCs treated with $\mathrm{Ac}_{4} \mathrm{ManNAz}(50 \mu \mathrm{M})$ and ADIBO-FITC $(40 \mu \mathrm{M})$ at 0,3 , and 5 days post incubation.

days after cell labeling the fluorescence decreased of about $30 \%$ according to the MSC growth rate. Importantly, the incubation of MSCs with $\mathrm{Ac}_{4} \mathrm{ManNAz}$ and ADIBO-FITC did not affect morphology and surface markers (Supporting Information Figure S2) and showed a $70 \pm 5 \%$ cells viability, measured by 3-(4,5-dimethylthiazol-2-yl)-2,5-diphenyltetrazolium bromide (MTT) assay, compared with not-treated control cells. In addition, treatment with $\mathrm{Ac}_{4} \mathrm{ManNAz}$ and $\mathrm{ADIBO}-\mathrm{FITC}$ did not alter the MSCs endocytic pathway as demonstrated by indirect immunofluorescence for specific molecules of different endocytic compartments. Figure 4 shows that MSCs with ADIBO-FITC with or without $\mathrm{Ac}_{4} \mathrm{ManNAz}$ have similar distribution and intensity of EEA1, Rab 5, that localize in early endosome, and they are involved in vesicle trafficking and Lamp-1 a lysosomal marker. Moreover, MSCs treated or not with $\mathrm{Ac}_{4} \mathrm{ManNAz}$ did not reveal significant changes of Tia-1 expression, that is, mRNA binding proteins that during stress conditions localize into stress granules.
Labeled MSCs Released Fluorescent EVs. Cell supernatants were collected from fluorescent $\mathrm{MSCs}$ ( $\mathrm{Ac}_{4} \mathrm{ManNAz}$ and ADIBO-FITC treated) cultured overnight in Roswell Park Memorial Institute (RPMI) without serum, for EVs purification. Labeled EVs were then obtained by ultracentrifugation. The advantage of the labeling protocol herein proposed is that unbound dyes are eliminated directly during MSC cells washing, and fluorescent exosomes are produced directly by cells and do not need any further purification. On the contrary, the procedure reported by Lee et al. ${ }^{29}$ was more laborious and required a purification through a PD SpinTrap G25 column. In particular, breast cancer cells (MCF7 and MDA-MB-231) were metabolically labeled with $\mathrm{Ac}_{4} \mathrm{ManNAz}$ and then exosomes were isolated by the cells and then labeled with the ADIBO-fluorescent dye molecules $\left(1 \mathrm{~h}\right.$ at $\left.37^{\circ} \mathrm{C}\right)$, and finally purified by gel filtration.

Labeled EVs were characterized by NanoSight analyses, Guava cytofluorimeter, and spectrofluorimetric analysis. EVs' number and size were quantified by nanoparticle tracking analysis (NTA). As shown in Figure 5A, the size distribution (nm) of EVs released by $\mathrm{Ac}_{4} \mathrm{ManNAz}$ and $\mathrm{ADIBO}-\mathrm{FITC}$ $(165.5 \pm 11 \mathrm{~nm})$ or ADIBO-FITC $(173 \pm 7 \mathrm{~nm})$ treated MSCs was similar to the one of nonlabeled EVs $(174 \pm 2 \mathrm{~nm})$, demonstrating that the ADIBO-FITC exposure on the EV membranes did not affect the EV size. Moreover, cytofluorimetric analyses, performed using Guava cytofluorimeter, of EVs released by $\mathrm{Ac}_{4} \mathrm{ManNAz}$ and ADIBO-FITC treated MSCs and by cells treated only with ADIBO-FITC, did not show any difference between the two EV types (Figure 5B). In particular, both EVs expressed several exosomal markers (Figure 5B) such as CD81, CD9, CD107, and CD63, known to be present on EVs and some mesenchymal stromal cells markers as CD73, CD44, and CD105, highly expressed on MSCs (Figure 5C). Moreover, both EVs showed the presence of the adhesion molecule CD29. It should be noted that MSC EVs enter into target cells by a receptor-mediated mechanism through CD44 and CD29 molecules, as previously demonstrated by Camussi et al. $^{30}$ Therefore, the presence of these molecules at a comparable level on EVs derived from $\mathrm{Ac}_{4} \mathrm{ManNAz}$ and ADIBO-FITC-treated MSCs and from cells treated only with ADIBO-FITC implies the retention of their excellent ability to enter into target cells.

Figure 6A shows the fluorescence intensity of FITC analyzed by Guava cytofluorimeter in unlabeled EVs derived from nontreated MSCs (control) compared with EVs produced after MSCs treatment with ADIBO-FITC or with $\mathrm{Ac}_{4} \mathrm{ManNAz}$ and

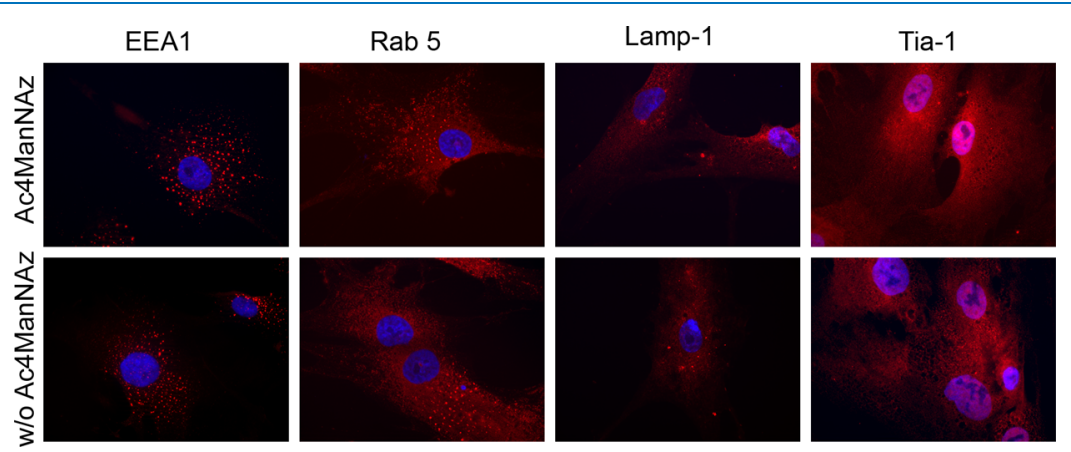

Figure 4. Immunofluorescence analysis of endocytic markers (EEA1, Rab5, Lamp-1, and Tia-1) on MSCs treated with ADIBO-FITC with or without $\mathrm{Ac}_{4}$ ManNaz. Nuclei were stained with Hoechst (blue). Marker fluorescence (red) was evaluated using a Zeiss LSM 5 Pascal model confocal microscope, magnification $63 \mathrm{x}$. 


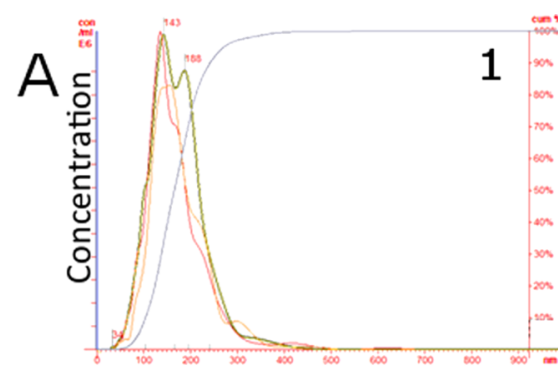

Particle Size

CD81

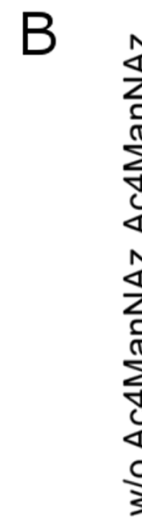

C
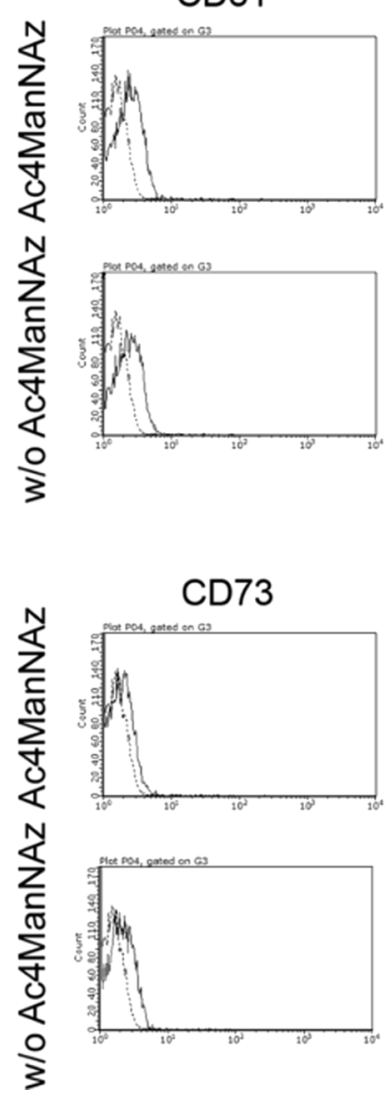

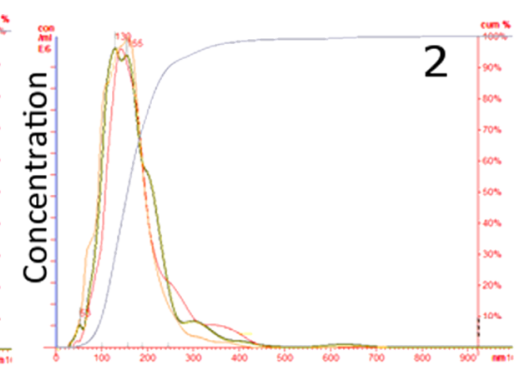

Particle Size

Exosomal markers
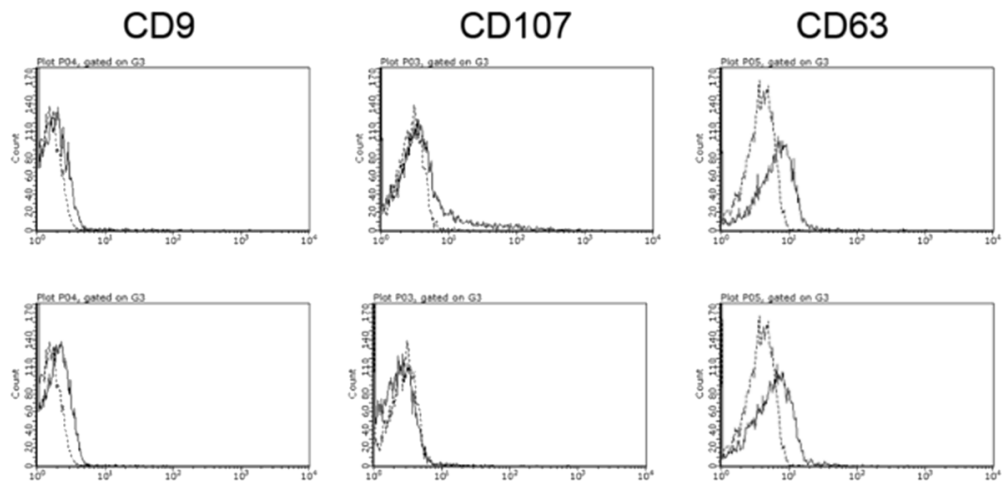

Mesenchymal markers
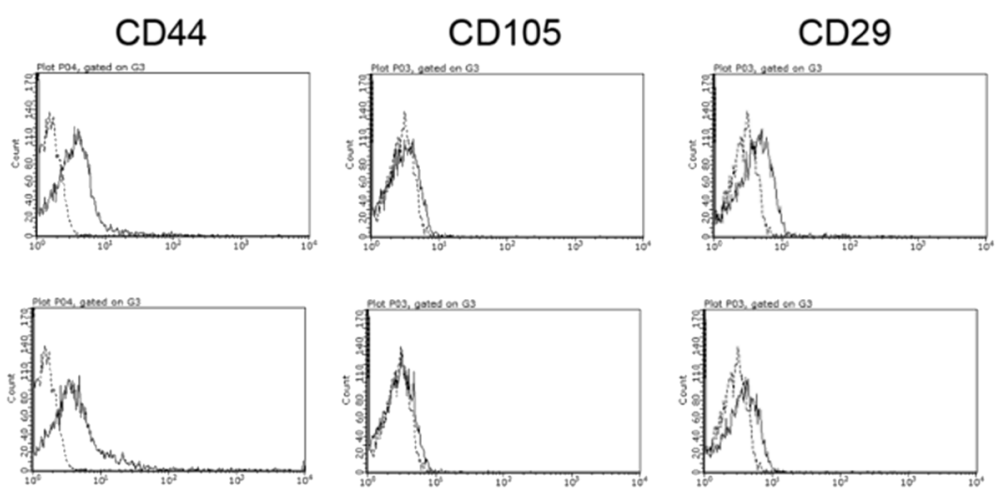

Figure 5. (A) Nanosight analysis. Representative size distribution of nonlabeled EVs (1), EVs derived from MCSs treated with ADIBO-FITC (2) or both $\mathrm{Ac}_{4} \mathrm{ManNAz}$ and $\mathrm{ADIBO}-\mathrm{FITC}$ (3). (B) Exosomal and (C) mesenchymal markers expressed on EVs labeled with ADIBO-FITC with or without $\mathrm{Ac}_{4} \mathrm{ManNAz}$.

ADIBO-FITC. The last EVs population showed a higher fluorescence intensity with respect to ADIBO-FITC-treated $\mathrm{EVs}$, thus demonstrating that the fluorescent dye introduced in the parent cells by the bio-orthogonal approach translates to a highly specific labeling of EVs. The level of different labeling of EVs was then also assessed by spectrofluorimetric measurements of their suspensions treated with triton $0.1 \%$ in phosphate-buffered saline (PBS) (Figure 6B). It is important to outline that the advantage of the herein proposed labeling protocol relies on the fact that the separation of unbound fluorescent dye occurs immediately after the cell incubation step, thus avoiding to carry out the ultracentrifugation of EVs in the presence of an excess of unbound dye. The unbound dye, being often highly hydrophobic, can form aggregates that can precipitate during the centrifugation step thus raising to an unspecific fluorescence.
Tracking of Labeled EVs in MSCs Cells. Finally, to assess whether the labeled EVs were still able to be uptaken into target MSCs, a dedicated incorporation assay was performed. Target cells were incubated for $1 \mathrm{~h}$ with EVs labeled as described above. Then, the cells were washed and the presence of fluorescent EVs within cells were evaluated by using a fluorescence microscope. As reported in Figure 7, EVs were clearly incorporated in the target cells generating a detectable fluorescent signal, definitely more intense in the case of $\mathrm{Ac}_{4} \mathrm{ManNAz}$ and ADIBO-FITC containing EVs thus confirming the validity of this labeling procedure based on the exploitation of a bio-orthogonal reaction. In conclusion, in the present study, a rapid, biocompatible, and specific chemical reaction between the FITC-functionalized cyclooctyne and azido-sugars, previously produced on the cell surface by metabolic glycoengineering, has been exploited to produce 

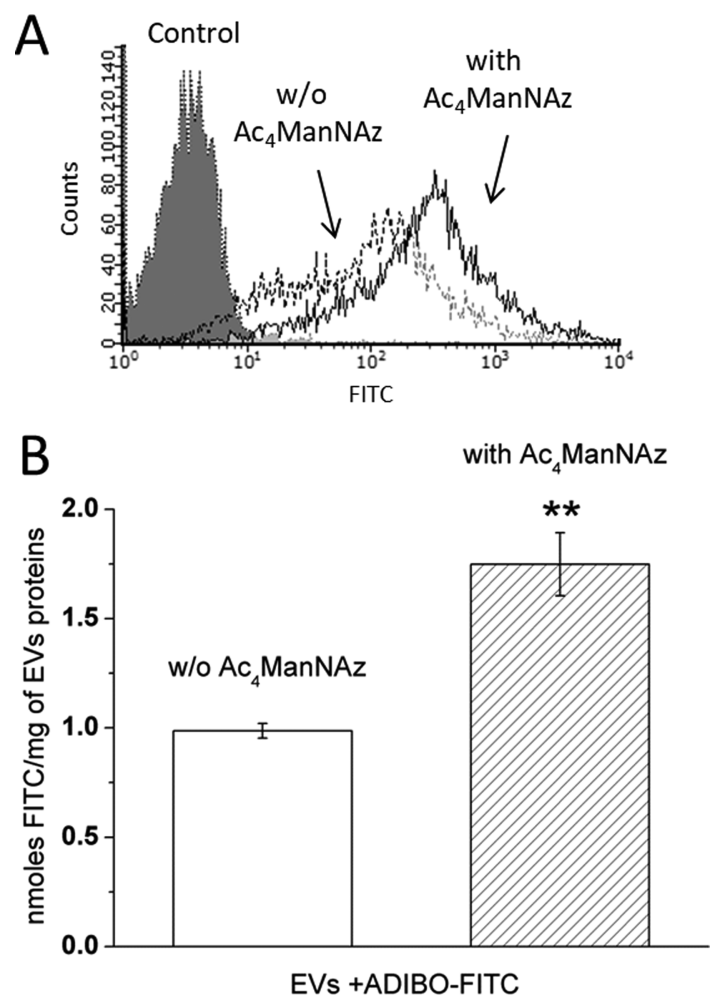

Figure 6. Representative cytofluorimetric (A) and spectrofluorimetric (B) analysis of EVs released by unlabeled MSCs (gray), and by MSCs labeled with ADIBO-FITC pre-incubated in the presence or in the absence of $\mathrm{Ac}_{4} \mathrm{ManNAz}$. Fluorescence values were normalized to the total EVs protein content determined by the Bradford assay. Data are the mean \pm SE of three different experiments. $* * p=0.0067$, Student $t$-test.
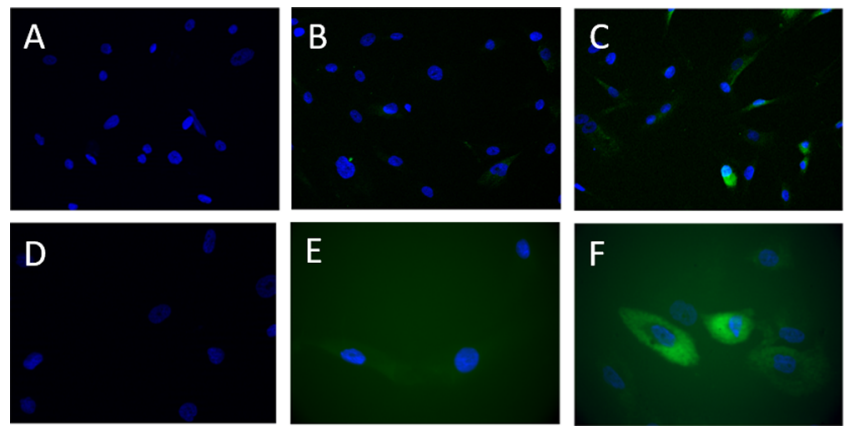

Figure 7. Fluorescent EVs internalization assay on MSCs. Cells were incubated in the presence of EVs released by MSCs untreated $(\mathrm{A}, \mathrm{D})$, treated with ADIBO-FITC (B,E) or $\mathrm{Ac}_{4} \mathrm{ManNAz}$ and ADIBO-FITC $(\mathrm{C}, \mathrm{F})$. Nuclei were stained with DAPI (blue). FITC fluorescence (green) was evaluated using an Apotome fluorescent microscope, magnification $20 \times(\mathrm{A}-\mathrm{C})$ and $40 \times(\mathrm{D}-\mathrm{F})$.

fluorescent exosomes. This approach aims at the development of an advanced strategy for direct and clean EVs labeling. The use of this labeling protocol can also be adapted to label EVs surface with drugs or other imaging agents that can be delivered to the pathological site, thanks to the specific recruitment properties of these EVs. Moreover, this method may allow the development of protocols for "in vivo" specific labeling of target cells with $\mathrm{Ac}_{4} \mathrm{ManNAz}$ followed by ADIBOdye/drug administration and thus tracking biodistribution and drug delivery.

\section{EXPERIMENTAL PROCEDURES}

All chemicals were purchased from Sigma-Aldrich or Alfa Aesar unless otherwise stated and were used without further purification.

The synthesis of $\mathrm{Ac}_{4} \mathrm{ManNAz}$ and $\mathrm{ADIBO}-\mathrm{FITC}$ was carried out following literature procedures. ${ }^{31}$ Briefly, $\mathrm{Ac}_{4} \mathrm{ManNAz}$ was synthesized by 1-[3-(dimethylamino)propyl]-3-ethylcarbodiimide hydrochloride and $N$-hydroxybenzotriazole mediated amidation of D-mannosamine hydrochloride with azidoacetic acid in methanol in the presence of triethylamine, followed by acetylation of the hydroxyl groups with acetic anhydride. Final $\mathrm{Ac}_{4} \mathrm{ManNAz}$ was obtained after column chromatographic purification in $90 \%$ overall yield.

ADIBO- $\mathrm{C}_{6}-\mathrm{NH}_{2}$ was synthesized starting from dibenzosuberenone by treatment with hydroxylamine to obtain dibenzosuberenone-oxime that was then rearranged to lactam by polyphosphoric acid-catalyzed Beckman rearrangement. The lactam was then reduced with $\mathrm{LiAlH}_{4}$ to give dihydrodibenzoazocine which was then acylated with 6(trifluoroacetamido)hexanoyl chloride. The alkyne was finally obtained via a bromination-dehydrobromination procedure to give the amino-functionalized aza-dibenzocyclooctyne (ADIBO- $\mathrm{C}_{6}-\mathrm{NH}_{2}$ ) after saponification of the trifluoroacetamide moiety with $\mathrm{K}_{2} \mathrm{CO}_{3}$ in aqueous methanol. The product ADIBO-FITC was synthesized by treating ADIBO- $\mathrm{C}_{6}-\mathrm{NH}_{2}$ with equimolar amounts of fluorescein isothiocyanate in dimethylformamide (DMF) in the presence of diisopropylethylamine (DIPEA) and finally purified by column chromatography.

Cell Labeling Experiments. Human bone marrow MSCs were purchased from Lonza and cultured and as previously described. ${ }^{32,33}$ MSCs derived from six preparations were used up to the seven passages of culture. MSCs were grown in T75 flasks and when they reached $80 \%$ confluence, they were incubated in the presence of $50 \mu \mathrm{M} \mathrm{Ac}{ }_{4} \mathrm{ManNAz}(0.25 \mathrm{v} / \mathrm{v} \%$ ethanol in MSC basal medium, Lonza) for $72 \mathrm{~h}$ at $37{ }^{\circ} \mathrm{C}, 5 \%$ $\mathrm{CO}_{2}$. After the incubation, they were washed three times with PBS and incubated for further $30^{\prime}$ at $37{ }^{\circ} \mathrm{C}, 5 \% \mathrm{CO}_{2}$ in the absence or in the presence of $40 \mu \mathrm{M}$ ADIBO-FITC $(0.2 \mathrm{v} / \mathrm{v} \%$ ethanol in MSC basal medium). Then, the cells were washed three times with PBS, detached with $1 \mathrm{mM}$ EDTA, resuspended in $250 \mu \mathrm{L}$ of PBS and analyzed for their FITC fluorescence and for mesenchymal stroma cell markers (CD 29, CD44, CD73, CD90, CD105, and CD146) by FACS. FACS analyses were performed immediately (day 0 ) or after 3 or 5 days post labeling using a BD FASCalibur, (Franklin Lakes, NY, USA); nontreated MSCs were used as control. Aliquots of the samples analyzed by FACS were exploited for total cell protein determination by the Bradford assay and spectrofluorometric analysis. Samples were sonicated in ice at $30 \%$ power for $30 \mathrm{~s}$ and after proteins determination they were diluted $1: 10$ in triton $0.1 \%$ in PBS and analyzed for their FITC fluorescence according to a FITC calibration curve (ex $492 \mathrm{~nm}$ em $517 \mathrm{~nm}$ in the range $0.5-30 \mathrm{nM})$. The obtained nanomoles of FITC calculated for each cell samples were normalized to milligrams of total cell proteins measured by Bradford assays.

Immunofluorescence Experiment. Immunofluorescence analysis of endocytic markers on MSCs treated with ADIBOFITC with or without $\mathrm{Ac}_{4} \mathrm{ManNAz}$ was performed on MSCs cultured on glass wells. MSCs were fixed in $4 \%$ paraformaldehyde containing $2 \%$ sucrose and permeabilized with HEPES- 
Triton X100 buffer (Sigma) immediately after the labeling procedure. The following primary antibodies (Santa Cruz) were used: EEA1 (sc-365652), Rab5 (sc-28570), Lamp-1 (sc8098), Tia-1(sc-1751). Omission of the primary antibodies was used as control. Alexa Fluor 596 anti-rabbit, or anti-goat or anti-mouse (Molecular Probes) were used as secondary antibodies. Confocal microscopy analysis was performed using a Zeiss LSM 5 Pascal model confocal microscope (Carl Zeiss International). Hoechst 33258 dye (Sigma) was added for nuclear staining.

MTT Assay. The MTT assay is based on the tetrazolium salts reduction to formazan by mitochondrial succinate dehydrogenase, which is quantified spectrophotometrically. MSCs cells previously treated with $50 \mu \mathrm{M} \mathrm{Ac} 4 \mathrm{ManNAz}_{4}$ for 72 $\mathrm{h}$ and $40 \mu \mathrm{M}$ ADIBO-FITC for $30^{\prime}$ were seeded at a density of $3 \times 10^{3}$ cells/well in a 96-well microtiter plate at $37^{\circ} \mathrm{C}$ and $5 \%$ $\mathrm{CO}_{2}$ air atmosphere for $24 \mathrm{~h}$. After this time, the medium was removed and $100 \mu \mathrm{L}$ of thiazolyl blue tetrazolium bromide dissolved in medium at the concentration of $0.45 \mathrm{mg} / \mathrm{mL}$ was added into each well and the plate was incubated for $4 \mathrm{~h}$ at 37 ${ }^{\circ} \mathrm{C}$ and $5 \% \mathrm{CO}_{2}$. Then, the medium was removed, $150 \mu \mathrm{L}$ of dimethyl sulfoxide was added into each well to solubilize the formazan salt crystals produced by the metabolism of live cells and the microplate was incubated at room temperature for 30 min. Finally, absorbance was read at $570 \mathrm{~nm}$ with iMark microplate reader (Biorad). Cell viability was reported as percentage of death cells observed in treated samples relative to that observed in not treated control cells. The experiment was performed in triplicate, and the data were presented as mean \pm SD

EVs Labeling Experiments. In agreement with the MSCs labeling procedure described above, the EVs were produced from MSCs cells pre-incubated with ADIBO-FITC in the presence or in the absence of $\mathrm{Ac}_{4} \mathrm{ManNAz}$. EVs were obtained from supernatants of MSCs cultured overnight in RPMI deprived of FCS. Cell supernatant was centrifuged at $3000 \mathrm{~g}$ for $20 \mathrm{~min}$ to remove cell debris and apoptotic bodies and subsequently was ultracentrifuged for $2 \mathrm{~h}$ at $100000 \mathrm{~g}$ (Beckman Coulter) as previously described. ${ }^{30}$

EVs Quantification. EVs were analyzed by NTA, using the NanoSight NS300 system (NanoSight Ltd, Amesbury, UK), equipped with a $405 \mathrm{~nm}$ laser and a high sensitivity digital camera system (OrcaFlash2.8, Hamamatsu C1 1440, Nanosight Ltd as previously described). ${ }^{33}$ For each sample, three videos of $30 \mathrm{~s}$ duration were recorded. The settings of acquisition and analysis were optimized and kept constant between samples. The final analysis reports the mean, mode, and median vesicle size and an estimation of the concentration.

EVs Cytofluorimetric Analysis. Cytofluorimetric analysis was performed using a Guava easyCyte flow cytometer (Millipore, Billerica, MA, USA) to detect EV fluorescence. In addition, the following PE-conjugated antibodies were used to characterize EV surface markers: anti-CD81, anti-CD9, antiCD107, anti-CD63, anti-CD73, anti-CD44, anti-CD105, and anti CD29 (all from Becton Dickinson). All experiments were analyzed with InCyte software.

EVs Spectrofluorometric Analysis. EVs were lysated in the presence of triton $0.1 \%$ in PBS and analyzed by spectrofluorimetry (HORIBA Jobin Yvon Fluoromax-4 spectrofluorometer, Edison, NY, USA). The nanomoles of FITC calculated for each EV samples were normalized to mg of total EVs proteins previously determined by the Bradford assay. EVs derived from not treated MSCs were used as control in all the performed studies.

Uptake of FITC-Labeled EVs on Target Cells. MSCs were cultured onto glass dishes and when they reached $80 \%$ confluence, they were incubated in the presence of 20000 FITC-labeled EVs/cell produced from MSCs pre-incubated with ADIBO-FITC in the presence or in the absence of $\mathrm{Ac}_{4}$ ManNAz. After $1 \mathrm{~h}$ of incubation at $37{ }^{\circ} \mathrm{C} 5 \% \mathrm{CO}_{2}$, the cells were extensively washed with PBS, they were fixed in PAF and nuclei were stained with DAPI (Sigma). Cells fluorescence was evaluated using an Apotome fluorescent microscope (Zeiss), magnification $20 \times$ and $40 \times$.

\section{ASSOCIATED CONTENT}

\section{Supporting Information}

The Supporting Information is available free of charge on the ACS Publications website at DOI: 10.1021/acsomega.8b00908.

Cytofluorimetric analysis of MSCs treated with $\mathrm{Ac}_{4} \mathrm{ManNaz}$ and different concentration of ADIBOFITC and of MSCs cell surface markers after treatment with $\mathrm{Ac}_{4} \mathrm{ManNAz}$ and ADIBO-FITC (PDF)

\section{AUTHOR INFORMATION}

\section{Corresponding Author}

*E-mail: simonetta.geninatti@unito.it (S.G.C.). ORCID

Lorenzo Tei: 0000-0002-7027-8396

Simonetta Geninatti Crich: 0000-0003-2998-5424

\section{Author Contributions}

D.A. and C.G. have contributed equally to this work.

Notes

The authors declare no competing financial interest.

\section{ACKNOWLEDGMENTS}

This project has received funding from by the AIRC investigator grant IG2013 no. 14565 and it was performed in the framework of the Consorzio CIRCMSB.

\section{ABBREVIATIONS}

$\mathrm{Ac}_{4} \mathrm{ManNAz}$, tetraacetylated $\mathrm{N}$-azidoacetyl-D-mannosamine; $\mathrm{ADIBO}$, aza-dibenzocyclooctyne; $\mathrm{CD}$, cluster of differentiation; $\mathrm{DiD}, 1,1$-dioctadecyl-3,3,3,3-tetramethylindodicarbocyanine; DiI, (1,1'-dioctadecyl-3,3,3',3'-tetramethylindocarbocyanine); DIPEA, diisopropylethylamine; DMF, dimethylformamide; EDTA, ethylenediaminetetraacetic acid; EEA1, early endosome antigen; EVs, extracellular vesicles; FACS, fluorescence activated cell sorting; FITC, fluorescein isothiocyanate; MSCs, mesenchymal stroma cells; MTT, 3-(4,5dimethylthiazol-2-yl)-2,5-diphenyltetrazolium bromide; NTA, nanoparticle tracking analysis; PBS, phosphate buffer saline; PKH, Paul Karl Horan; R18, octadecyl rhodamine B chloride; RPMI, Roswell Park Memorial Institute

\section{REFERENCES}

(1) Ratajczak, M. Z.; Ratajczak, J. Extracellular Microvesicles as Game Changers in Better Understanding the Complexity of Cellular Interactions-From Bench to Clinical Applications. Am. J. Med. Sci. 2017, 354, 449-452.

(2) Camussi, G.; Deregibus, M. C.; Cantaluppi, V. Role of stem-cellderived microvesicles in the paracrine action of stem cells: Figure 1. Biochem. Soc. Trans. 2013, 41, 283-287. 
(3) Ratajczak, M. Z.; Ratajczak, J. Horizontal transfer of RNA and proteins between cells by extracellular microvesicles: 14 years later. Clin. Transl. Med. 2016, 5, 7.

(4) Ratajczak, J.; Miekus, K.; Kucia, M.; Zhang, J.; Reca, R.; Dvorak, P.; Ratajczak, M. Z. Embryonic stem cell-derived microvesicles reprogram hematopoietic progenitors: evidence for horizontal transfer of mRNA and protein delivery. Leukemia 2006, 20, 847-856.

(5) Valadi, H.; Ekström, K.; Bossios, A.; Sjöstrand, M.; Lee, J. J.; Lötvall, J. O. Exosome-mediated transfer of mRNAs and microRNAs is a novel mechanism of genetic exchange between cells. Nat. Cell Biol. 2007, 9, 654-659.

(6) Derkus, B.; Emregul, K. C.; Emregul, E. A new approach in stem cell research-Exosomes: Their mechanism of action via cellular pathways. Cell Biol. Int. 2017, 41, 466-475.

(7) Bruno, S.; Porta, S.; Bussolati, B. Extracellular vesicles in renal tissue damage and regeneration. Eur. J. Pharmacol. 2016, 790, 83-91.

(8) Zhan, C.; Ma, C.-b.; Yuan, H.-m.; Cao, B.-y.; Zhu, J.-j. Macrophage-derived microvesicles promote proliferation and migration of Schwann cell on peripheral nerve repair. Biochem. Biophys. Res. Commun. 2015, 468, 343-348.

(9) Gai, C.; Carpanetto, A.; Deregibus, M. C.; Camussi, G. Extracellular vesicle-mediated modulation of angiogenesis. Histol. Histopathol. 2016, 31, 379-391.

(10) Grange, C.; Iampietro, C.; Bussolati, B. Stem cell extracellular vesicles and kidney injury. Stem Cell Invest. 2017, 4, 90.

(11) Giebel, B.; Kordelas, L.; Börger, V. Clinical potential of mesenchymal stem/stromal cell-derived extracellular vesicles. Stem Cell Invest. 2017, 4, 84.

(12) Lai, R. C.; Yeo, R. W. Y.; Lim, S. K. Mesenchymal stem cell exosomes. Semin. Cell Dev. Biol. 2015, 40, 82-88.

(13) Wallace, P. K.; Tario, J. D., Jr.; Fisher, J. L.; Wallace, S. S.; Ernstoff, M. S.; Muirhead, K. A. Tracking antigen-driven responses by flow cytometry: monitoring proliferation by dye dilution. Cytometry, Part A 2008, 73A, 1019-1034.

(14) Grange, C.; Tapparo, M.; Bruno, S.; Chatterjee, D.; Quesenberry, P. J.; Tetta, C.; Camussi, G. Biodistribution of mesenchymal stem cell-derived extracellular vesicles in a model of acute kidney injury monitored by optical imaging. Int. J. Mol. Med. 2014, 33, 1055-1063.

(15) Montecalvo, A.; Larregina, A. T.; Shufesky, W. J.; Stolz, D. B.; Sullivan, M. L. G.; Karlsson, J. M.; Baty, C. J.; Gibson, G. A.; Erdos, G.; Wang, Z.; Milosevic, J.; Tkacheva, O. A.; Divito, S. J.; Jordan, R.; Lyons-Weiler, J.; Watkins, S. C.; Morelli, A. E. Mechanism of transfer of functional microRNAs between mouse dendritic cells via exosomes. Blood 2012, 119, 756-766.

(16) Dominkuš, P. P.; Stenovec, M.; Sitar, S.; Lasič, E.; Zorec, R.; Plemenitaš, A.; Žagar, E.; Kreft, M.; Lenassi, M. PKH26 labeling of extracellular vesicles: Characterization and cellular internalization of contaminating PKH26 nanoparticles. Biochim. Biophys. Acta, Biomembr. 2018, 1850, 1350-1361.

(17) Stremersch, S.; Brans, T.; Braeckmans, K.; De Smedt, S.; Raemdonck, K. Nucleic acid loading and fluorescent labeling of isolated extracellular vesicles requires adequate purification. Int. J. Pharm. 2017, DOI: 10.1016/j.ijpharm.2017.10.022.

(18) Chang, P. V.; Prescher, J. A.; Sletten, E. M.; Baskin, J. M.; Miller, I. A.; Agard, N. J.; Lo, A.; Bertozzi, C. R. Copper-free click chemistry in living animals. Proc. Natl. Acad. Sci. U.S.A. 2010, 107, $1821-1826$.

(19) Sletten, E. M.; Bertozzi, C. R. Bioorthogonal chemistry: fishing for selectivity in a sea of functionality. Angew. Chem., Int. Ed. 2009, 48, 6974-6998.

(20) Ramil, C. P.; Lin, Q. Bioorthogonal Chemistry: strategies and recent developments. Chem. Commun. 2013, 49, 11007-11022.

(21) Neef, A. B.; Schultz, C. Selective Fluorescence Labeling of Lipids in Living Cells. Angew. Chem., Int. Ed. 2009, 48, 1498-1500.

(22) Yoon, H. Y.; Koo, H.; Kim, K.; Kwon, I. C. Molecular imaging based on metabolic glycoengineering and bioorthogonal click chemistry. Biomaterials 2017, 132, 28-36.
(23) Yoon, H. I.; Yhee, J. Y.; Na, J. H.; Lee, S.; Lee, H.; Kang, S.-W.; Chang, H.; Ryu, J. H.; Lee, S.; Kwon, I. C.; Cho, Y. W.; Kim, K. Bioorthogonal copper free click chemistry for labeling and tracking of chondrocytes in vivo. Bioconjugate Chem. 2016, 27, 927-936.

(24) Kang, S.-W.; Lee, S.; Na, J. H.; Yoon, H. I.; Lee, D.-E.; Koo, H.; Cho, Y. W.; Kim, S. H.; Jeong, S. Y.; Kwon, I. C.; Choi, K.; Kim, K. Cell Labeling and Tracking Method without Distorted Signals by Phagocytosis of Macrophages. Theranostics 2014, 4, 420-431.

(25) Neves, A. A.; Wainman, Y. A.; Wright, A.; Kettunen, M. I.; Rodrigues, T. B.; McGuire, S.; Hu, D.-E.; Bulat, F.; Crich, S. G.; Stöckmann, H.; Leeper, F. J.; Brindle, K. M. Imaging Glycosylation In Vivo by Metabolic Labeling and Magnetic Resonance Imaging. Angew. Chem. 2016, 128, 1308-1312.

(26) Lee, S.; Yoon, H. I.; Na, J. H.; Jeon, S.; Lim, S.; Koo, H.; Han, S.-S.; Kang, S.-W.; Park, S.-J.; Moon, S.-H.; Park, J. H.; Cho, Y. W.; Kim, B.-S.; Kim, S. K.; Lee, T.; Kim, D.; Lee, S.; Pomper, M. G.; Kwon, I. C.; Kim, K. In vivo stem cell tracking with imageable nanoparticles that bind bioorthogonal chemical receptors on the stem cell surface. Biomaterials 2017, 139, 12-29.

(27) Xie, R.; Dong, L.; Du, Y.; Zhu, Y.; Hua, R.; Zhang, C.; Chen, X. In vivo metabolic labeling of sialoglycans in the mouse brain by using a liposome-assisted bioorthogonal reporter strategy. Proc. Natl. Acad. Sci. U.S.A. 2016, 113, 5173-5178.

(28) Laughlin, S. T.; Agard, N. J.; Baskin, J. M.; Carrico, I. S.; Chang, P. V.; Ganguli, A. S.; Hangauer, M. J.; Lo, A.; Prescher, J. A.; Bertozzi, C. R. Metabolic labeling of glycans with azido sugars for visualization and glycoproteomics. Methods Enzymol. 2006, 415, 230-250.

(29) Lee, T. S.; Kim, Y.; Zhang, W.; Song, I. H.; Tung, C.-H. Facile metabolic glycan labeling strategy for exosome tracking. Biochim. Biophys. Acta, Gen. Subj. 2018, 1862, 1091-1100.

(30) Bruno, S.; Grange, C.; Deregibus, M. C.; Calogero, R. A.; Saviozzi, S.; Collino, F.; Morando, L.; Busca, A.; Falda, M.; Bussolati, B.; Tetta, C.; Camussi, G. Mesenchymal stem cell-derived microvesicles protect against acute tubular injury. J. Am. Soc. Nephrol. 2009, 20, 1053-1067.

(31) Kuzmin, A.; Poloukhtine, A.; Wolfert, M. A.; Popik, V. V. Surface Functionalization Using Catalyst-Free Azide-Alkyne Cycloaddition. Bioconjugate Chem. 2010, 21, 2076-2085.

(32) Bruno, S.; Collino, F.; Deregibus, M. C.; Grange, C.; Tetta, C.; Camussi, G. Microvesicles derived from human bone marrow mesenchymal stem cells inhibit tumor growth. Stem Cells Dev. 2013, 22, 758-771.

(33) Bruno, S.; Tapparo, M.; Collino, F.; Chiabotto, G.; Deregibus, M. C.; Lindoso, R. S.; Neri, F.; Kholia, S.; Giunti, S.; Wen, S.; Quesenberry, P.; Camussi, G. Renal Regenerative Potential of Different Extracellular Vesicle Populations Derived from Bone Marrow Mesenchymal Stromal Cells. Tissue Eng., Part A 2017, 23, $1262-1273$. 